JIRSS (2018)

Vol. 17, No. 02, pp 141-164

DOI: $10.29252 /$ jirss.17.2.7

\title{
A New Distribution Family Constructed by Fractional Polyno- mial Rank Transmutation
}

\author{
Mehmet Yilmaz \\ Ankara University, Faculty of Science, Department of Statistics, Ankara, Turkey.
}

Received: 21/07/2017, Revision received: 07/12/2017, Published online: 07/08/2018

\begin{abstract}
In this study, a new polynomial rank transmutation is proposed with the help of the idea of quadratic rank transmutation mapping (QRTM). This polynomial rank transmutation is allowed to extend the range of the transmutation parameter from $[-1,1]$ to $[-1, k]$. At this point, the generated distributions gain more flexibility than a transmuted distribution constructed by QRTM. The distribution family obtained in this transmutation is considered to be an alternative to the distribution families obtained by quadratic rank transmutation. Statistical and reliability properties of this family are examined. Considering Weibull distribution as the base distribution, the importance and the flexibility of the proposed families are illustrated by two applications.
\end{abstract}

Keywords. Polynomial Rank Transmutation, Quadratic Rank Transmutation, Transmuted Distribution, Transmuted-Weibull.

MSC: 60E05; 62E15.

\section{Introduction}

Numerous studies have been conducted by many authors using the quadratic rank transformation proposed by Shaw and Buckley (2007). According to QRTM, the c.d.f

Mehmet Yilmaz (mehmetyilmaz@ankara.edu.tr) 
$\mathrm{H}$ of the generated distribution corresponding to the baseline distribution having c.d.f $\mathrm{F}$ is given by $H=F+\lambda F(1-F)=(1+\lambda) F-\lambda F^{2}$, where $-1 \leq \lambda \leq 1$. However, many other distributions have been derived with the help of families such as Marshall-Olkin generated family (MO-G) by Marshall and Olkin (1997), beta generated family by Eugene et al. (2002), transformed-transformer family by Alzaatreh et al. (2013) and Weibull-G by Bourguignon et al. (2014). In recent years, studies have focused on the generalized G-families and the generalized transmuted families. Some of these studies can be given as follows. Yousof et al. (2015) introduced the transmuted exponentiated generalized-G family. The beta transmuted- $\mathrm{H}$ family was introduced by Afify et al. (2017). Korkmaz and Genc (2017) considered the standard two-sided power distribution to define classes like the beta- $G$ and the Kumaraswamy-G classes. Cordeiro et al. (2017) proposed a new class of continuous distributions with two extra shape parameters named the generalized odd log-logistic family of distributions. Alizadeh et al. (2017) introduced a new transmuted G-family of distribution. Nofal et al. (2017) introduced a three-parameter transmuted class.

The overall objective is the success of the generated distributions in real-data modeling. We present a transformation that can be obtained by the convex combination of the distributions of order statistics, which is similar to the polynomial rank transmutation proposed by Shaw and Buckley (2007). We will discuss it in detail in Section 2.

\section{Motivation}

We express the idea behind the definition of quadratic rank transmutation, which is introduced by Shaw and Buckley (2007) in Subsection 4.2, and then move to the occurrence of numerous studies as follows. Let us consider two-component systems (series and parallel) that component lifetime is distributed as $F$. Then distributions of the lifetimes of series and parallel systems, respectively, are $2 F-F^{2}$ and $F^{2}$. Note that $F$ lies between these two distribution functions, namely, the ordering amongst three distribution functions is given by

$$
F^{2} \leq F \leq 2 F-F^{2}
$$

Accordingly, $F$ can be represented by a convex combination of $2 F-F^{2}$ and $F^{2}$ as $\delta\left(2 F-F^{2}\right)+(1-\delta) F^{2}$, where $\delta$ is a convex combination parameter belonging to the 
interval $[0,1]$. In particular, $F$ is obtained if delta is taken as $\frac{1}{2}$. In addition, it is possible to obtain numerous distribution functions. Let us call the distribution function obtained by this convex transformation as $H$. Then $H$ can be represented by the following form:

$$
H=2 \delta F-(2 \delta-1) F^{2} .
$$

By re-parameterizing $2 \delta-1=\lambda$, where $\lambda \in[-1,1], H$ becomes $(1+\lambda) F-\lambda F^{2}$. Thus, we achieve the quadratic rank transmutation given in equation (48) by Shaw and Buckley (2007). A similar result was obtained independently by Mirhossaini and Dolati (2008), where $H$ represents the mixing of the extreme order statistic of a random sample of size two.

In this paper we propose a new rank transmutation in the light of the idea behind the works of Shaw and Buckley (2007) and Mirhossaini and Dolati (2008). Let $F_{r: n}$ be a distribution of the $r^{\text {th }}$ order statistic obtained from a sample of size $n$ from a population whose distribution is $F$. Then $F_{r: n}$ is represented as a tail probability of a binomial distribution with $F$ as the probability of success and $n$ as the number of trials. Thus, $F_{r: n}$ can be written according to the equation (2.2.13) by Arnold et al. (2008) as follows:

$$
F_{r: n}(x)=\sum_{j=r}^{n}\left(\begin{array}{c}
n \\
j
\end{array}\right) F^{j}(x) \bar{F}^{n-j}(x),
$$

where $\bar{F}$ denotes the survival function. Hence, inequality (2.1) can be re-written by this notation as $F_{2: 2} \leq F_{1: 1} \leq F_{1: 2}$. By taking into account this ordering properties, it is obvious that $F_{k+1: k+1} \leq F_{k: k+1}$ holds for $k=1,2, \ldots, n-1$, where $F_{k+1: k+1}=F^{k+1}$ and $F_{k: k+1}=(k+1) F^{k}-k F^{k+1}$. Furthermore, $F_{k: k}$ lies between $F_{k+1: k+1}$ and $F_{k: k+1}$. In other words, we have

$$
F_{k+1: k+1} \leq F_{k: k} \leq F_{k: k+1} \text {. }
$$

Then, any distribution that lies between $F_{k+1: k+1}$ and $F_{k: k+1}$ can be represented by a convex combination of $F_{k+1: k+1}$ and $F_{k: k+1}$ as $F_{k: k}$. Let $H$ stand for this distribution. Then, for $\delta \in[0,1]$, we have

$$
H=\delta F_{k: k+1}+(1-\delta) F_{k+1: k+1} .
$$

Interestingly, another way of obtaining bounds for $F_{k: k}$ is to multiply both sides of the equation (2.1) by $F^{k-1}$. Hence, we have another ordering as

$$
F_{k+1: k+1} \leq F_{k: k} \leq F^{k-1}\left(2 F-F^{2}\right) \text {. }
$$


Similarly, if a convex combination of $F_{k+1: k+1}$ and $F^{k-1}\left(2 F-F^{2}\right)$ is represented by $H^{*}$, for $\delta^{*} \in[0,1]$, we have

$$
H^{*}=\delta^{*} F^{k-1}\left(2 F-F^{2}\right)+\left(1-\delta^{*}\right) F_{k+1: k+1} .
$$

Many distributions can be generated by using both equations (2.4) and (2.6). Specially for $\delta=\frac{1}{k+1}$ and $\delta^{*}=\frac{1}{2}$ we achieve $H=H^{*}=F_{k: k}$. Furthermore, for $k=1$ we have $H=H^{*}=F$. This leads us to define a new rank transmutation mapping as in the following section. However, these generate many distributions and, since $F^{k-1}\left(2 F-F^{2}\right) \leq F_{k: k+1}, H$ has a wider range than $H^{*}$ in terms of the distribution functions generated by that. This leads to the convex combination parameter of $H$ giving more flexibility than the parameter of $H^{*}$. For this reason, $H$ is more attractive to us.

\section{Construction of the New Family of Distribution}

Let us start with rearranging the equation (2.4). Then we have

$$
H=(k+1) \delta F^{k}-(-1+(k+1) \delta) F^{k+1} .
$$

If $(-1+(k+1) \delta)$ is reparemetrized as $\lambda$, where the range of $\lambda$ is $[-1, k]$, equation (3.1) can be represented by

$$
H=(1+\lambda) F^{k}-\lambda F^{k+1} .
$$

We observe that, for $k=1, H$ indicates the well-known quadratic rank transmuted distribution. For $\lambda=0$, we have the baseline distribution as the distribution of the maximum of $k$ independent copies of $F$. For $\lambda=-1$, we have the failure distribution of the lifetime of the parallel system consisting of $k+1$ independent components. Namely, $H$ is the distribution of the lifetime of the system defined as $T=\max \left\{X_{1}, \cdots, X_{k+1}\right\}$. Here, component lifetimes, namely $X_{j}$ 's, are independent and distributed as $F$. For $\lambda=1$, where $k \geq 1$, the $\mathrm{cdf} H$ indicates that the failure probability of the system lifetime is defined as $T=\max \left\{X_{1}, \cdots, X_{k-1}, \min \left\{X_{k}, X_{k+1}\right\}\right\}$. For $\lambda=k, H$ indicates a failure probability of $k-o u t-o f-k+1$ system lifetime. This proposed rank transmutation mapping allows to extend the range of the transmutation parameter from $[-1,1]$ to $[-1, k]$. At this point, generated distributions gain more flexibility. Similarly, making some rearrangements on equation $(2.6), H^{*}$ is represented by

$$
H^{*}=2 \delta^{*} F^{k}-\left(-1+2 \delta^{*}\right) F^{k+1},
$$


and, by taking $\left(-1+2 \delta^{*}=\lambda\right)$ for $\lambda \in[-1,1]$, we have

$$
H^{*}=(1+\lambda) F^{k}-\lambda F^{k+1} \text {. }
$$

Hence, $H^{*}$ can be viewed as a special case of $H$. So, we will only deal with the distribution family $H$ in the subsequent parts of the study.

\section{Representations of cdf, pdf and the Survival Function}

Since we are using order statistics while constructing this new distribution, $k$ is always considered to be a nonnegative integer number. However, the value of $k$ is not limited to integers, but considering $k \geq 1$ is a sufficient assumption for defining a distribution. We will discuss this in terms of the pdf of the distribution. $H(t)$ can be represented by

$$
\begin{aligned}
H(t) & =\delta F_{k: k+1}(t)+(1-\delta) F_{k+1: k+1}(t) \\
& =(1+\lambda) F^{k}(t)-\lambda F^{k+1}(t) \\
& =F^{k}(t)[1+\lambda \bar{F}(t)] \\
& =(1+\lambda) F_{k: k}(t)-\lambda F_{k+1: k+1}(t),
\end{aligned}
$$

where $\delta \in[0,1]$ and $\lambda \in[-1, k]$. Specially, note that $F$ indicates the baseline distribution when $k=1$, and $\lambda=0$, otherwise, for $k \geq 1, F^{k}$ is considered as a baseline distribution at $\lambda=0$. The corresponding pdf is given by

$$
\begin{aligned}
h(t) & =f(t) F^{k-1}(t)[(1+\lambda) k-\lambda(k+1) F(t)] \\
& =f(t) F^{k-1}(t)[\lambda k \bar{F}(t)+k-\lambda F(t)] \\
& =(1+\lambda) f_{k: k}(t)-\lambda f_{k+1: k+1}(t) .
\end{aligned}
$$

As seen immediately from the second equality in (4.2), $h(t), k \geq 1$ provides $h(t) \geq 0$. Hence the transmutation parameter set is defined as $\{(\lambda, k): k \in[1, \infty), \lambda \in[-1, k]\}$. We can also express the series expansion of $h(t)$ for future discussions by applying the generalized binomial expansion. Then we have

$$
\begin{aligned}
h(t) & =f(t)\left[\sum_{j=0}^{\infty}\left(\begin{array}{c}
k-1 \\
j
\end{array}\right)(-1)^{j} \bar{F}(t)^{j}\right][\lambda k \bar{F}(t)+k-\lambda F(t)] \\
& =f(t)\left[\lambda(1+k) \sum_{j=0}^{\infty}\left(\begin{array}{c}
k-1 \\
j
\end{array}\right)(-1)^{j} \bar{F}(t)^{j+1}+(k-\lambda) \sum_{j=0}^{\infty}\left(\begin{array}{c}
k-1 \\
j
\end{array}\right)(-1)^{j} \bar{F}(t)^{j}\right] .
\end{aligned}
$$


Now, focusing on the polynomial structure given in equation (66) in Shaw and Buckley (2007), we call the distribution generated by $H$ as fractional polynomial transmuted distribution, shortly as FPT $-D$. According to the equation (4.1), the survival function of the FPT-random variable $T$ is given by

$$
\begin{aligned}
\bar{H}(t) & =1-F^{k}(t)[1+\lambda \bar{F}(t)] \\
& =(1+\lambda) \bar{F}_{k: k}(t)-\lambda \bar{F}_{k+1: k+1}(t) .
\end{aligned}
$$

\section{Moment generating function and raw moments of FPT-random variable}

In this section, we shall present the moment generating function and sth raw moments of the FPT-random variable

$$
M_{T}(v)=(1+\lambda) M_{X_{k: k}}(v)-\lambda M_{X_{k+1: k+1}}(v)
$$

and

$$
E\left[T^{s}\right]=(1+\lambda) E\left[X_{k: k}^{s}\right]-\lambda E\left[X_{k+1: k+1}^{s}\right]
$$

Note that, these two expressions are valid for integer $k$.

\section{Hazard Rate and Reversed Hazard Rate Functions of FPT- Random Variable}

From equation (4.3), hazard rate function denoted by $r(t)$ can be defined by

$$
r(t)=\frac{-d \log (\bar{H}(t))}{d t}=w_{1}(t) r_{F_{k: k}}(t)+w_{2}(t) r_{F_{k+1: k+1}}(t),
$$

where $w_{1}(t)=\frac{(1+\lambda) \bar{F}_{k: k}(t)}{(1+\lambda) \bar{F}_{k: k}(t)-\lambda \bar{F}_{k+1: k+1}(t)}$ and $w_{2}(t)=1-w_{1}(t)$. Component hazard rates are, respectively, given by

$$
r_{F_{k: k}}(t)=k r_{F}(t) \frac{F^{k-1}(t) \bar{F}(t)}{1-F^{k}(t)}, \quad r_{F_{k+1: k+1}}(t)=(k+1) r_{F}(t) \frac{F^{k}(t) \bar{F}(t)}{1-F^{k+1}(t)} .
$$


The reversed hazard rate function, $r v(t)$, can be obtained by using the third equality in (4.1) as

$$
r v(t)=\frac{d \log (H(t))}{d t}=r v_{F}(t)\left[1+k-\frac{1+\lambda}{1+\lambda-\lambda F(t)}\right] .
$$

It can be seen that $r v(t)$ increases in $t$ for $\lambda \in[-1,0]$ if $r v_{F}(t)$ increases in $t$, and for $\lambda>0, r v(t)$ decreases in $t$ for $\lambda \in[-1,0]$ if $r v_{F}(t)$ decreases in $t$. Furthermore, $r(t)$ also increases in $t$ for $\lambda \in[-1,0]$, since $r v(t)\left[-1+\frac{1}{\bar{H}(t)}\right]=r(t)$.

\section{Generating Random Number from FPT-D}

The number generation will be done with convex combination notation given in the first equality of the equation (4.1). At this point, it is possible to describe the $F P T-D$ as a 2-component mixture distribution, and the number generation will be easier then.

The first algorithm is considered for integer values of $k$.

- Step 1: Generate $k+1$ random numbers from the baseline distribution, namely $X_{1}, X_{2}, \ldots, X_{k+1} \sim F$, and sort them in ascending order.

- Step 2: Generate a random number $U$ from the uniform distribution on $(0,1)$.

- Step 3: If $U \leq\left(\frac{1+\lambda}{k+1}\right)$, a random number $T$ from FPT-D is $k$-th smallest value of $X_{1}, X_{2}, \ldots, X_{k+1}$. Namely, $T=X_{k: k+1}$. Otherwise, $T=F^{-1}\left(U^{\frac{1}{k+1}}\right)$ or $T=X_{k+1: k+1}$.

The second algorithm is considered for $k \geq 1$.

- Step 1: Generate a random number $U$ from a uniform distribution on $(0,1)$.

- Step 2: If $U \leq\left(\frac{1+\lambda}{k+1}\right)$, a random number $T$ from FPT-D is obtained as the solution of the equation as $v^{k}(k+1-k v)-U=0$. Thus, $T=F^{-1}(v)$. Otherwise, $T=F^{-1}\left(U \frac{1}{k+1}\right)$.

In the next section, specific distributions chosen for this family will be considered to show usefulness of the model on a real data application. Note that the mathematical or statistical characterizations of these specific distributions will not be included because of the lack of space. 


\section{ML Estimations of Ransmutation Parameters}

In this section, we discuss only about how to estimate $k$ and $\lambda$ parameters of the FPT-D. Here, since the parameter $\lambda$ functionally depends on $k$, we will consider the convex combination expression for $h(t)$ to simplify maximization of the log-likelihood. By considering (4.1), let $f_{1}$ and $f_{2}$ denote the corresponding pdf of $F_{k: k+1}$ and $F_{k+1: k+1}$. Then we have

$$
\begin{aligned}
h(t) & =\delta_{1} f_{1}(t)+\delta_{2} f_{2}(t)=\delta_{1}\left(k(k+1) f(t) F^{k-1}(t) \bar{F}(t)\right)+\delta_{2}\left((k+1) f(t) F^{k}(t)\right) \\
& =(k+1) f(t) F^{k-1}(t)\left(\delta_{1} k \bar{F}(t)+\delta_{2} F(t)\right),
\end{aligned}
$$

where $\delta_{1}+\delta_{2}=1$. Let $T_{1}, T_{2}, \cdots, T_{n}$ be i.i.d random variables of a sample size $n$ from the FPT-D. Then, according to (8.1), constrained $\log$-likelihood $\ell=\log L(k, \lambda ; \Theta, t)$ can be written as

$$
\begin{aligned}
\ell= & n \log (1+k)+(k-1) \sum_{j=1}^{n} \log \left(F\left(t_{j} ; \Theta\right)\right)+\sum_{j=1}^{n} \log \left(f\left(t_{j} ; \Theta\right)\right) \\
& +\sum_{j=1}^{n} \log \left(\delta k+(1-\delta(1+k)) F\left(t_{j} ; \Theta\right)\right)-\epsilon\left(\delta_{1}+\delta_{2}-1\right),
\end{aligned}
$$

where $\Theta$ is the parameter set of $F$. According to partial derivatives with respect to $k$, $\delta_{1}, \delta_{2}$ and $\epsilon$, likelihood equations are given by

$$
\begin{aligned}
& \frac{\partial \ell}{\partial k}=\frac{n}{1+k}+\sum_{j=1}^{n} \log \left(F\left(t_{j} ; \Theta\right)\right)+\sum_{j=1}^{n} \frac{\delta \bar{F}\left(t_{j} ; \Theta\right)}{\left(\delta k+(1-\delta(1+k)) F\left(t_{j} ; \Theta\right)\right)}=0, \\
& \frac{\partial \ell}{\partial \delta_{1}}=\sum_{j=1}^{n} \frac{f_{1}\left(t_{j} ; \Theta\right)}{\left(\delta_{1} F_{1}\left(t_{j} ; \Theta\right)+\delta_{2} F_{2}\left(t_{j} ; \Theta\right)\right)}-\epsilon=0, \\
& \frac{\partial \ell}{\partial \delta_{2}}=\sum_{j=1}^{n} \frac{f_{2}\left(t_{j} ; \Theta\right)}{\left(\delta_{1} F_{1}\left(t_{j} ; \Theta\right)+\delta_{2} F_{2}\left(t_{j} ; \Theta\right)\right)}-\epsilon=0, \\
& \text { and } \\
& \frac{\partial \ell}{\partial \epsilon}=\delta_{1}+\delta_{2}-1=0 .
\end{aligned}
$$


From the last three equations, we have an iterative solution for $\delta$ as given by

$$
\delta^{(1)}=\frac{1}{n} \sum_{j=1}^{n} \frac{\delta^{(0)} k \bar{F}\left(t_{j} ; \Theta\right)}{\left(\delta^{(0)} k \bar{F}\left(t_{j} ; \Theta\right)+\left(1-\delta^{(0)}\right) F\left(t_{j} ; \Theta\right)\right)} .
$$

The iterative solution of parameter $k$ is obtained by the first likelihood equation assuming $\delta$ as $\delta^{(0)}$.

The steps are repeated until the absolute difference of log likelihood is small. Then, ML estimates of transmutation parameters are obtained. To show that ML equation for $k$ has at least one solution, it is useful to give the following proposition.

Proposition 8.1. If $\frac{n}{2} \geq-\sum_{j=1}^{n} \log \left(F\left(t_{j} ; \Theta\right)\right)$, then the equation $\partial \ell / \partial k=0$ has at least one root in $[1, \infty)$, where $\delta$ is the true value of the parameter.

Proof. Let $\omega(k)$ denote the function on the RHS of the expression $\partial \ell / \partial k$, then it is clear that

$$
\lim _{k \rightarrow \infty} \omega(k)=\sum_{j=1}^{n} \log \left(F\left(t_{j} ; \Theta\right)\right)<0,
$$

and

$$
\begin{aligned}
\lim _{k \rightarrow 1} \omega(k)= & \frac{n}{2}+\sum_{j=1}^{n} \log \left(F\left(t_{j} ; \Theta\right)\right)+\sum_{j=1}^{n} \frac{\delta \bar{F}\left(t_{j} ; \Theta\right)}{\left(\delta \tilde{F}\left(t_{j} ; \Theta\right)+(1-\delta) F\left(t_{j} ; \Theta\right)\right)} \\
& \geq \frac{n}{2}+\sum_{j=1}^{n} \log \left(F\left(t_{j} ; \Theta\right)\right) \geq 0 .
\end{aligned}
$$

In the next section, the Weibull distribution is chosen for this family. We examine some statistical properties for FPT-Weibull distribution, specifically.

\section{Special Case: FPT-Weibull Distribution}

The Weibull distribution is widely used in reliability and lifetime data analysis due to its flexibility. The values of the shape and the scale parameters affect on distributional characteristics such as the shape of the pdf curve, the reliability and the hazard rate. For 
this reason, the specific base distribution is taken as Weibull. The following notation is considered to represent Weibull pdf, which is given by

$$
f(t ; \sigma, \eta)=\frac{\eta}{\sigma}\left(\frac{t}{\sigma}\right)^{\eta-1} e^{-\left(\frac{t}{\sigma}\right)^{\eta}}, t>0, \sigma>0, \eta>0
$$

We will investigate the pdf of the FPT-Weibull distribution with some possible shapes of the parameter set $(\sigma, \eta, k, \lambda)$. Then, we obtain a series expansion representation of raw moments, mean, variance, skewness and kurtosis measures. Finally, the simulation results for estimating the parameters are given.

\subsection{Pdf of FPT-Weibull distribution}

According to (4.2) and (4.3), the pdf of the FPT-Weibull distribution can be represented by

$$
\begin{aligned}
h(t)= & \frac{\eta}{\sigma}\left(\frac{t}{\sigma}\right)^{\eta-1} e^{-\left(\frac{t}{\sigma}\right)^{\eta}}\left(1-e^{-\left(\frac{t}{\sigma}\right)^{\eta}}\right)^{k-1}\left[(k-\lambda)+\lambda(k+1) e^{-\left(\frac{t}{\sigma}\right)^{\eta}}\right] \\
= & (k-\lambda) \frac{\eta}{\sigma}\left(\frac{t}{\sigma}\right)^{\eta-1} \sum_{j=0}^{\infty}\left(\begin{array}{c}
k-1 \\
j
\end{array}\right)(-1)^{j} e^{-\left(\frac{t}{\sigma}\right)^{\eta}(j+1)} \\
& +\lambda(k+1) \frac{\eta}{\sigma}\left(\frac{t}{\sigma}\right)^{\eta-1} \sum_{j=0}^{\infty}\left(\begin{array}{c}
k-1 \\
j
\end{array}\right)(-1)^{j} e^{-\left(\frac{t}{\sigma}\right)^{\eta}(j+2)} .
\end{aligned}
$$

Figure 1 illustrates some of the possible shapes of the pdf of a FPT-Weibull random variable for selected values of the parameters $\eta, k$ and $\lambda$ for $\sigma=1$.

\subsection{Raw moments, mean, variance, skewness and kurtosis of FPT-Weibull random variables}

Firstly, the raw moments of the FPT-Weibull random variable were obtained from

Nadarajah et al. (2012). Inspired by studies on the exponentiated Weibull distribution 

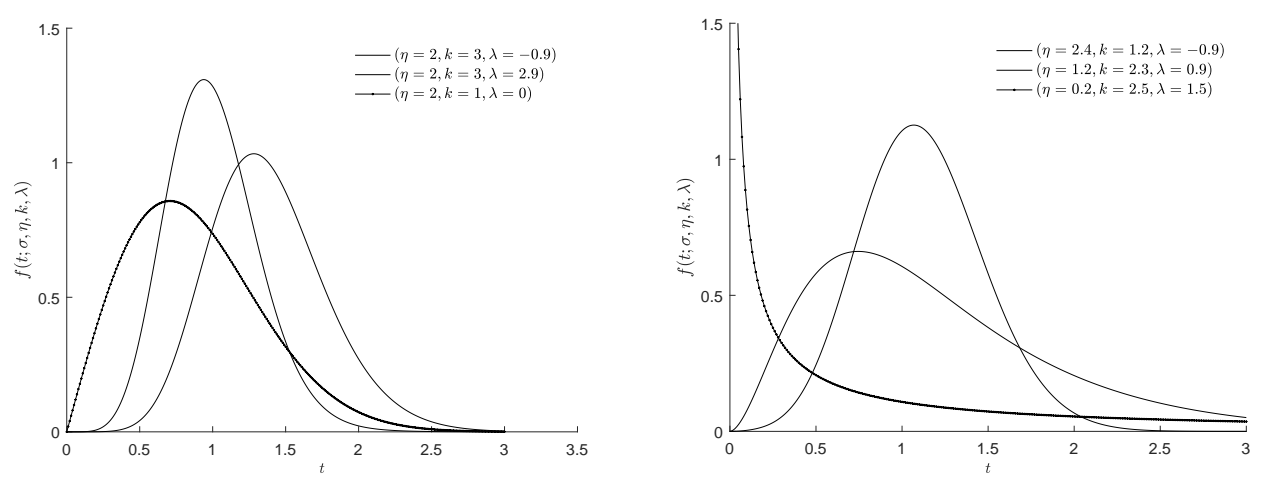

Figure 1: Some possible shapes of pdf of FPT-Weibull random variable for $\sigma=1$.

and taking into account (9.1), the $m$ th raw moments are given by

$$
\begin{aligned}
E\left[T^{m}\right]= & (k-\lambda) \sigma^{m} \Gamma\left(\frac{m}{\eta}+1\right) \sum_{j=0}^{\infty}\left(\begin{array}{c}
k-1 \\
j
\end{array}\right)(-1)^{j}(1+j)^{-\left(\frac{m}{\eta}+1\right)} \\
& +\lambda(k+1) \sigma^{m} \Gamma\left(\frac{m}{\eta}+1\right) \sum_{j=0}^{\infty}\left(\begin{array}{c}
k-1 \\
j
\end{array}\right)(-1)^{j}(2+j)^{-\left(\frac{m}{\eta}+1\right)} \\
= & \sigma^{m} \Gamma\left(\frac{m}{\eta}+1\right) \sum_{j=0}^{\infty}\left(\begin{array}{c}
k-1 \\
j
\end{array}\right)(-1)^{j}\left(\begin{array}{c}
(k-\lambda)(1+j)^{-\left(\frac{m}{\eta}+1\right)} \\
+\lambda(k+1)(2+j)^{-\left(\frac{m}{\eta}+1\right)}
\end{array}\right) .
\end{aligned}
$$

Hence, Figure 2 indicates how the first moment changes with $\lambda$ for $\sigma=1$ and $k=3$. By using (9.2), as we have no implicit formula for the variance, the variance of the FPT-Weibull random variable can be represented as Figure 3.

Based on the first three moments of the FPT-Weibull random variable, the skewness measure of this random variable can be calculated by

$$
\gamma_{1}=\frac{E[T-E(T)]^{3}}{\operatorname{Var}(T)^{3 / 2}}
$$

According to Rousu (1973) and (9.2), the skewness measure of Weibull random variables is given by

$$
\gamma_{1}=\frac{\Gamma\left(\frac{3}{\eta}+1\right)-3 \Gamma\left(\frac{2}{\eta}+1\right) \Gamma\left(\frac{1}{\eta}+1\right)+2 \Gamma\left(\frac{1}{\eta}+1\right)^{3}}{\left[\Gamma\left(\frac{2}{\eta}+1\right)-\Gamma\left(\frac{1}{\eta}+1\right)^{2}\right]^{3 / 2}}
$$



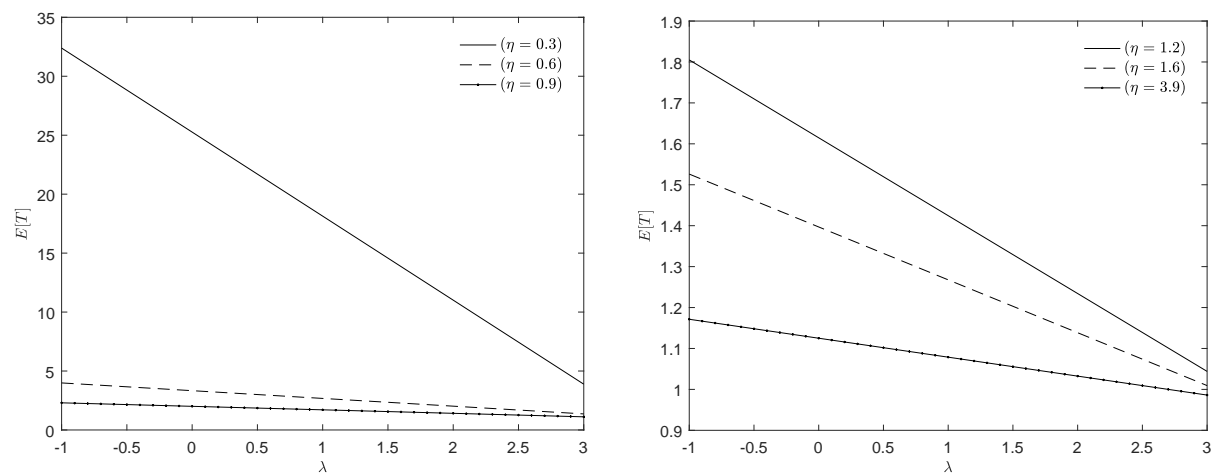

Figure 2: Some possible shapes of first moment of FPT-Weibull random variable w.r.t to $\lambda$ for $\sigma=1$ and $k=3$.

Table 1: Skewness measure of Weibull distribution for some values of $\eta$.

\begin{tabular}{|c|c|c|c|c|c|c|}
\hline$\eta$ & 0.6 & 0.9 & 1.2 & 1.4 & 2.6 & 3.9 \\
\hline$\gamma_{1}$ & 4.59 & 2.35 & 1.52 & 1.20 & 0.32 & -0.07 \\
\hline
\end{tabular}

The tabulated values of the skewness measure of Weibull distribution for some values of $\eta$ are given in Table 1. Afterwards, shapes of skewness measure for FPTWeibull random variable are given in Figure 4.

Based on the first four moments of the FPT-Weibull random variable, the kurtosis measure of this random variable can be calculated by

$$
\gamma_{2}=\frac{E[T-E(T)]^{4}}{\operatorname{Var}(T)^{2}}
$$

According to Rousu (1973) and (9.2), the kurtosis measure of Weibull random variable is given by

$$
\gamma_{2}=\frac{\Gamma\left(\frac{4}{\eta}+1\right)-4 \Gamma\left(\frac{3}{\eta}+1\right) \Gamma\left(\frac{1}{\eta}+1\right)+6 \Gamma\left(\frac{2}{\eta}+1\right) \Gamma\left(\frac{1}{\eta}+1\right)^{2}-3 \Gamma\left(\frac{1}{\eta}+1\right)^{4}}{\left[\Gamma\left(\frac{2}{\eta}+1\right)-\Gamma\left(\frac{1}{\eta}+1\right)^{2}\right]^{2}} .
$$

Tabulated values of the kurtosis measure of Weibull distribution for some values of $\eta$ are given in Table 2. Afterwards, shapes of skewness measure for FPT-Weibull random variable are given in Figure 5. 

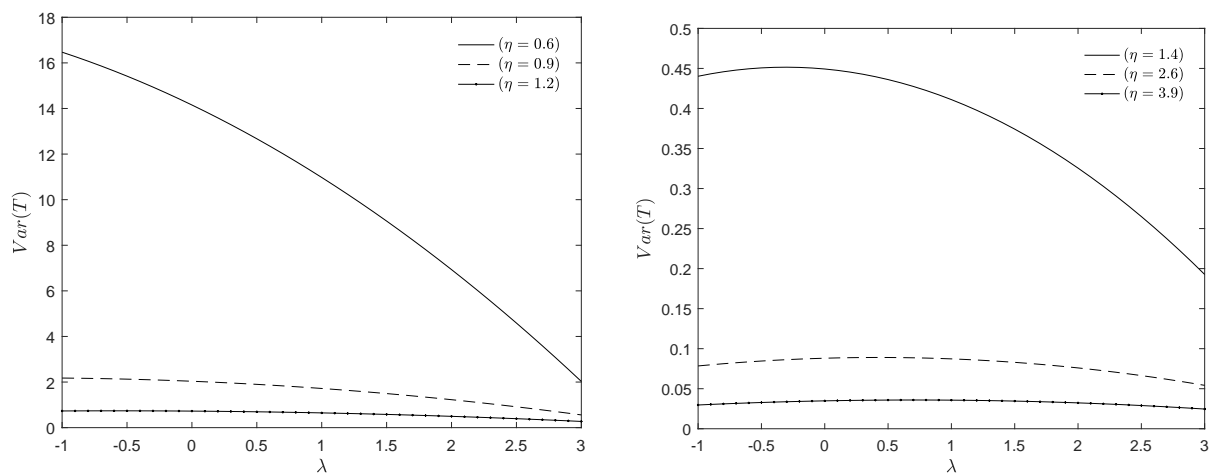

Figure 3: Some possible shapes of variance of FPT-Weibull random variable w.r.t to $\lambda$ for $\sigma=1$ and $k=3$.

Table 2: Kurtosis measure of Weibull distribution for some values of $\eta$.

\begin{tabular}{|c|c|c|c|c|c|c|}
\hline$\eta$ & 0.6 & 0.9 & 1.2 & 1.4 & 2.6 & 3.9 \\
\hline$\gamma_{2}$ & 40.691 & 12.833 & 10.313 & 12.128 & 71.465 & 297.362 \\
\hline
\end{tabular}

As the value of $\eta$ increases, FPT-Weibull distribution is less flattened than the Weibull distribution.

\subsection{Hazard rate function of FPT-Weibull}

From equation (6.1), the hazard rate function of the FPT-Weibull is given by

$$
r(t)=\frac{\eta}{\sigma}\left(\frac{t}{\sigma}\right)^{\eta-1} \frac{\left(1-e^{-\left(\frac{t}{\sigma}\right)^{\eta}}\right)^{k-1} e^{-\left(\frac{t}{\sigma}\right)^{\eta}}\left[k-\lambda+\lambda(1+k) e^{-\left(\frac{t}{\sigma}\right)^{\eta}}\right]}{1-(1+\lambda)\left(1-e^{-\left(\frac{t}{\sigma}\right)^{\eta}}\right)^{k}+\lambda\left(1-e^{-\left(\frac{t}{\sigma}\right)^{\eta}}\right)^{k+1}} .
$$

Some shapes of the hazard rate function of the FPT-Weibull random variable are given in Figure 6. The first three graphs, respectively, show the shapes of hazard rate functions of Weibull, transmuted-Weibull and FPT-Weibull. 

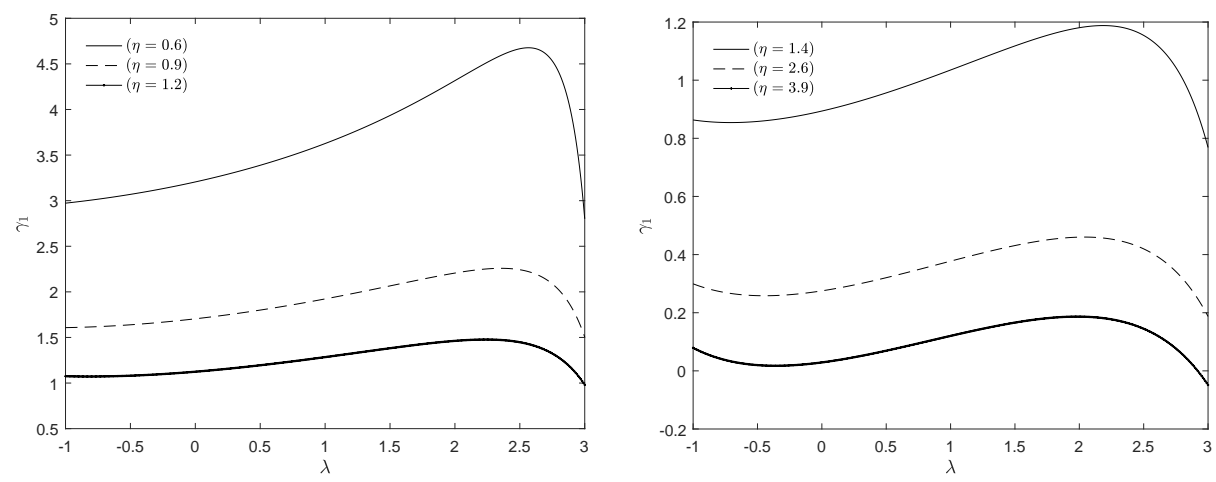

Figure 4: Some possible shapes of skewness measure of FPT-Weibull random variable w.r.t to $\lambda$ for $\sigma=1$ and $k=3$.

\subsection{Simulation study for ML estimates of the transmutation parameters of FPT-Weibull}

We conduct the simulation study to evaluate the performance of MLEs of the $k$ and $\lambda$ with respect to the sample size for FPT-Weibull distribution. All results are obtained from 1000 Monte Carlo replications, and the simulations are carried out using Matlab software. According to the second algorithm given in Section 7, we generate samples from the FPT-Weibull distribution for different sizes $\mathrm{n}=20,50,100$ for the fixed choice of the parameters $\{k=1.2, \lambda=0.6\},\{k=2, \lambda=0.8\},\{k=5, \lambda=3.8\}$ and $\{k=8, \lambda=6\}$, where $\sigma=1$ and $\eta=2.4$. Table 3 describes the results for different parameter values with their corresponding root mean squared errors (RMSE).

According to Table 3, the bias of estimates increases while the values of $k$ and $\lambda$ increase. Furthermore, the standard errors of $\lambda$ estimates are higher than in $k$ for each sample size.

\section{Application}

In this section, two applications based on two real data sets are presented to demonstrate the modeling performance of the FPT-Weibull distributions. We compare the FPTWeibull with Weibull and transmuted Weibull (Aryal and Tsokos , 2011) distributions. Throughout the application section, we use the Akaike Information Criterion (AIC) as a model selection criterion. Besides, Kolmogorov-Smirnov, Anderson-Darling and 

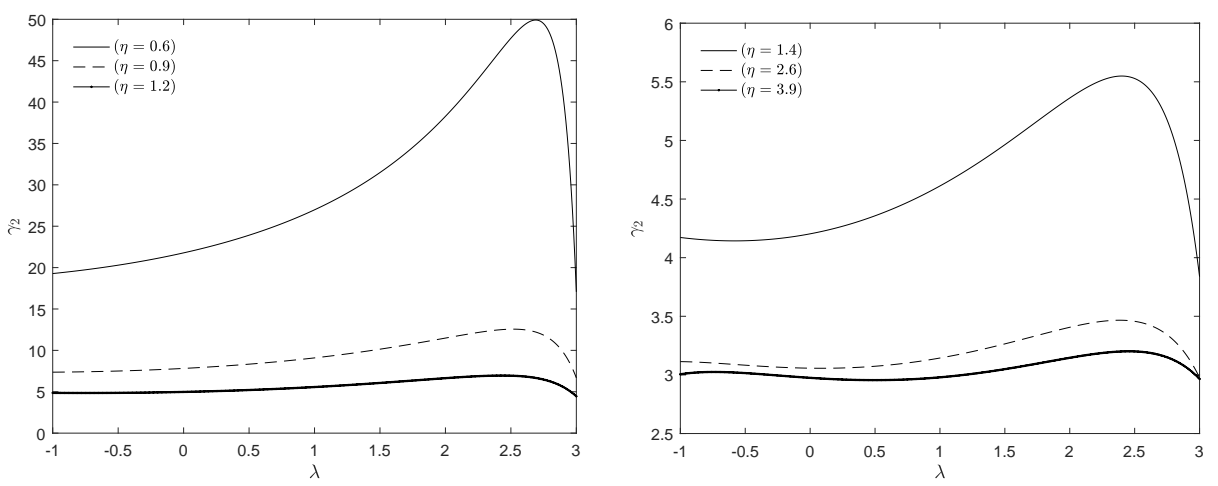

Figure 5: Some possible shapes of kurtosis measure of FPT-Weibull random variable w.r.t to $\lambda$ for $\sigma=1$ and $k=3$.

Cramér-von Mises statistics are taken into account as measures of goodness-of-fit.

\section{Akaike information criterion:}

$$
A I C=-2 \log L(\Theta ; \underset{\sim}{x})+2 m,
$$

where $m$ is the size of the parameter vector $\Theta$.

\section{Goodness-of-fit statistics}

- Kolmogorov- Smirnov

$$
k-S=\sup _{x}\left|H(x)-H_{n}(x)\right|,
$$

where $H_{n}(x)$ is the empirical distribution function.

\section{- Anderson-Darling}

$$
\mathcal{A}^{2}=-n-\frac{1}{n} \sum_{j=1}^{n}\left\{(2 j-1) \log \left[H\left(t_{(j)} ; \hat{\Theta}\right)\right]+(2(n-j)+1) \log \left[1-H\left(t_{(j)} ; \hat{\Theta}\right)\right]\right\} \text {. }
$$

(see, Stephens (1979), Anderson (2011) and Pogány et al. (2015)). 

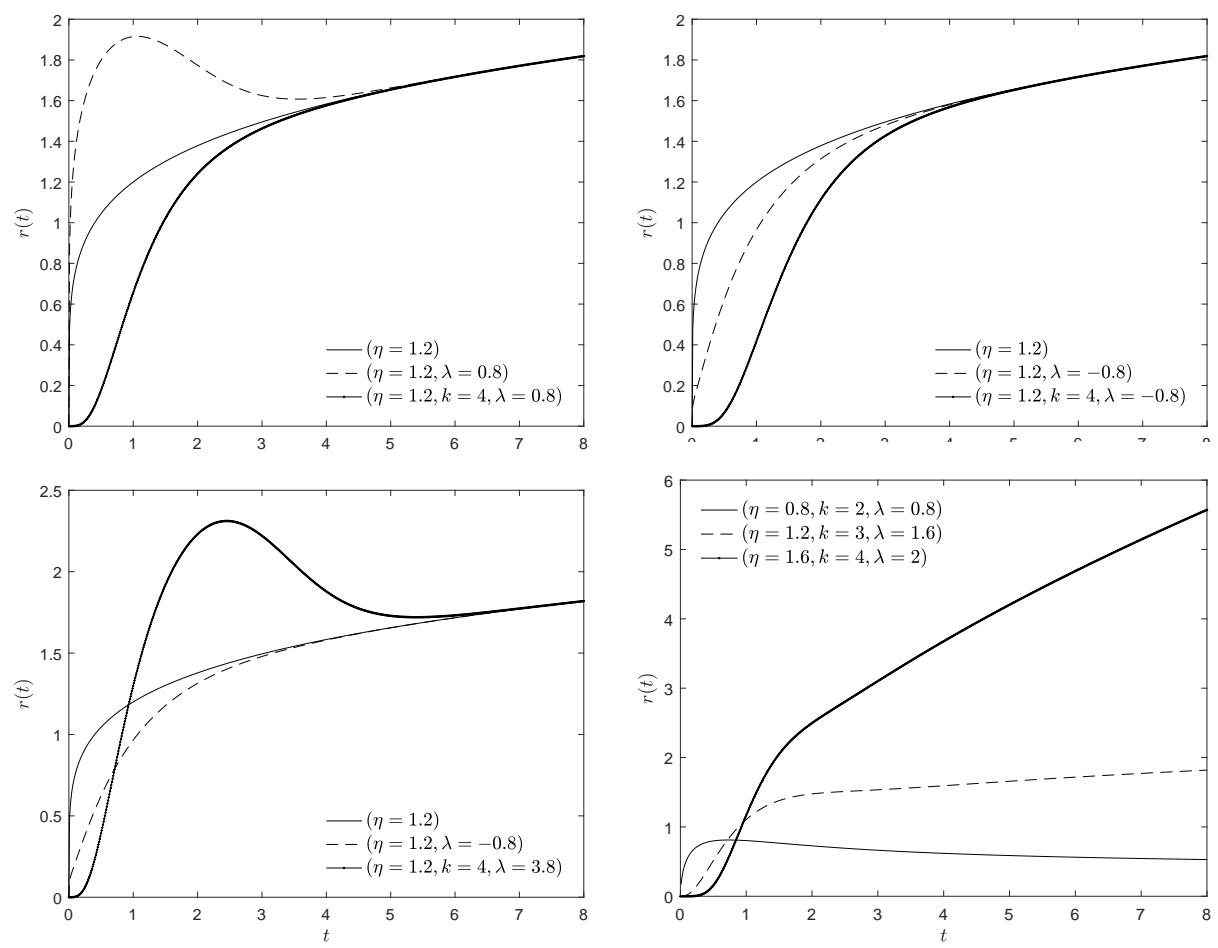

Figure 6: Some possible shapes of hazard rate function of FPT-Weibull random variable for $\sigma=1$.

- Cramér-von Mises

$$
W^{2}=\frac{1}{12 n}+\sum_{j=1}^{n}\left(H\left(t_{(j)} ; \hat{\Theta}\right)-\frac{2 j-1}{2 n}\right)^{2},
$$

where $t_{(j)}$ is the jth ordered sample, $t_{(1)} \leq \cdots \leq t_{(j-1)} \leq t_{(j)} \leq \cdots \leq t_{(n)}$ (see, Pogány et al. (2015)).

Data set 1: The first data set contains survival times (in days) of Guinea pigs injected with different doses of tubercle bacilli and is given in Table 4 . The data has been analyzed by Singh et al. (2013).

The Weibull, transmuted Weibull (T-Weibull) and polynomial transmuted Weibull (FPT-Weibull) distributions are fitted to the data and the MLEs of the parameters are computed. Values of Kolmogorov-Smirnov statistic (K-S), Akaike information criterion 
Table 3: Monte Carlo simulation results based on MLE and RMSE for the transmutation parameters of FPT- Weibull distribution.

\begin{tabular}{cccc}
\hline \multirow{2}{*}{ Parameters } & \multicolumn{3}{c}{ Sample Size, $n$} \\
\cline { 2 - 4 } & 20 & 50 & 100 \\
\hline$k=1.2$ & $1.3004(0.3408)$ & $1.2435(0.2157)$ & $1.2197(0.1588)$ \\
$\lambda=0.6$ & $0.6650(0.4361)$ & $0.6378(0.2963)$ & $0.6180(0.2227)$ \\
\hline$k=2$ & $2.1429(0.6918)$ & $2.0339(0.4707)$ & $2.0292(0.3380)$ \\
$\lambda=0.8$ & $0.9223(0.7748)$ & $0.8334(0.5899)$ & $0.8136(0.4231)$ \\
\hline$k=5$ & $5.1337(1.2483)$ & $5.0278(0.8140)$ & $5.0349(0.5693)$ \\
$\lambda=3.8$ & $3.7531(1.4185)$ & $3.7443(1.0181)$ & $3.8209(0.6934)$ \\
\hline$k=8$ & $6.9977(1.9909)$ & $7.3819(1.6185)$ & $7.7499(1.1865)$ \\
$\lambda=6$ & $4.3259(3.9380)$ & $4.9708(3.0979)$ & $5.5620(2.1492)$ \\
\hline
\end{tabular}

(AIC), Anderson-Darling statistic $\left(\mathcal{A}^{2}\right)$ and Cramér-von Mises statistic $\left(\mathcal{W}^{2}\right)$ are also given with these MLEs of the parameters in Table 5. A graphical comparison of the fitted models is displayed in Figure 7 and Figure 8. Results show that the FPT -Weibull distribution fits best to the Guinea pigs data, having the smallest goodness of fit statistics among all three candidate distributions. In order to visually show the performance of this model, the histogram, Q-Q and P-P graphs are also given in Figure 8.

According to P-P plots in Figure 8, we plotted $F_{W}\left(x_{(i)}, \hat{\sigma}, \hat{\eta}\right), F_{T-W}\left(x_{(i)}, \hat{\sigma}, \hat{\eta}, \hat{\lambda}\right)$ and $F_{F P T-W}\left(x_{(i)}, \hat{\sigma}, \hat{\eta}, \hat{\lambda}, \hat{k}\right)$ against $\frac{i-.375}{n+.25}$, where $x_{(i)}$ 's are the ordered values of the Guinea pigs data. We also calculate the coefficient of determination, $R^{2}$, for each model. These values, respectively, are $0.9084,0.9300$ and 0.9783 . It is again seen that the FPT-Weibull model fits better than the other two models.

According to Figure 7 and Figure 8, FPT -Weibull distribution shows a reasonably good fit, especially in the right tail area. Fitting right tail area is particularly important for many life testing reliability problems.

Data set 2: The second data set is about the remission times (in months) of a random 
Table 4: Survival times (in days) of Guinea pigs injected with different doses of tubercle bacilli.

\begin{tabular}{llllllllllll}
\hline 0.012 & 0.015 & 0.022 & 0.024 & 0.032 & 0.033 & 0.034 & 0.038 & 0.043 & 0.044 & 0.048 & 0.052 \\
0.053 & 0.054 & 0.055 & 0.056 & 0.057 & 0.058 & 0.059 & 0.060 & 0.061 & 0.062 & 0.063 & 0.065 \\
0.067 & 0.068 & 0.070 & 0.072 & 0.073 & 0.075 & 0.076 & 0.081 & 0.083 & 0.084 & 0.085 & 0.087 \\
0.091 & 0.095 & 0.096 & 0.098 & 0.099 & 0.109 & 0.110 & 0.121 & 0.127 & 0.129 & 0.131 & 0.143 \\
0.146 & 0.175 & 0.211 & 0.233 & 0.258 & 0.263 & 0.297 & 0.341 & 0.376 & 0.030 & 0.036 & 0.043 \\
0.061 & 0.060 & 0.063 & 0.063 & 0.063 & 0.072 & 0.081 & 0.153 & 0.181 & 0.260 & 0.347 & 0.074 \\
\hline
\end{tabular}

sample of 128 bladder cancer patients. This data set, taken from Lee and Wang (2003), is given in Table 6.

We fit Weibull, T-Weibull, and FPT-Weibull distributions to this data set. the MLEs of the parameters, the values of Kolmogorov-Smirnov statistic (K-S), Akaike information criterion (AIC), Anderson-Darling statistic $\left(\mathcal{A}^{2}\right)$ and Cramér-von Mises statistic $\left(\mathcal{W}^{2}\right)$ are given in Table 7. A graphical comparison of the fitted models is displayed in Figure 9 and Figure 10.

It is clear from Table 7 that based on K-S, AIC, $\mathcal{A}^{2}$ and $\mathcal{W}^{2}$ the proposed FPTWeibull model provides a better fit than the other two models to this data set. The relative histogram and the fitted pdf of the models are plotted in Figure 9. In order to visually show the performance of the FPT-Weibull model for the bladder patients' data, the histogram, Q-Q and P-P graphs are also given in Figure 10. The calculated values of coefficient of determination for each model, respectively, are $0.9836,0.9912$ and 0.9972 .

\section{Conclusion}

In this paper, we proposed and studied a new class of distributions called the Fractional Polynomial Transmuted Family (FPT-D). This polynomial transmutation led the range of transmutation parameter to extend from $[-1,1]$ to $[-1, k]$. We investigate several structural properties such as the cumulative distribution function, the probability density function, moment generating function, raw moments, survival and hazard functions. Obviously, this extension provides a little flexibility to analyze real life data. Two examples of real data empirically proved the importance and potentiality of the 
Table 5: MLEs of the model parameters and values of goodness of fit statistics for models (Guinea pigs data).

\begin{tabular}{|c|cc|c|c|c|c|}
\hline Model & \multicolumn{2}{|c|}{ Parameter Estimates } & K-S & AIC & $\mathcal{A}^{2}$ & $\mathcal{W}^{2}$ \\
\hline Weibull & $\hat{\sigma}=0.1118$ & $\hat{\eta}=1.4058$ & 0.1527 & -195.68 & 2.4518 & 0.4484 \\
\hline T- Weibull & $\begin{array}{r}\hat{\sigma}=0.1412 \\
\hat{\lambda}=0.5992\end{array}$ & 0.1350 & -197.17 & 2.0330 & 0.3577 \\
\hline FPT -Weibull & $\begin{array}{r}\hat{\sigma}=0.0151 \\
\hat{\lambda}=8.0436\end{array} \quad \hat{k}=0.5532$ & $\mathbf{0 . 0 8 6 6}$ & $\mathbf{- 2 0 6 . 5 6}$ & $\mathbf{0 . 7 0 9 9}$ & $\mathbf{0 . 1 2 5 6}$ \\
\hline
\end{tabular}

proposed family. The FPT-Weibull provided better fits than Weibull and transmuted Weibull distributions when applying to real data sets. According to ML estimates of transmutation parameters in illustrative examples, these are both greater than 1 . Therefore, extended range of the transmutation parameter can be more useful among other transmuted candidates for modeling.

Acknowledgments. The author would like to thank the anonymous referees for their constructive comments and suggestions helping us to improve the manuscript.

\section{References}

Afify, A. Z., Yousof, H. M. and Nadarajah, S. (2017), The beta transmuted-H family for lifetime data. Statistics and Its Interface, 10(3), 505-520.

Alizadeh, M., Merovci, F. and Hamedani, G. G. (2017), Generalized transmuted family of distributions: properties and applications. Hacettepe Journal of Mathematics and Statistics, 46(4), 645-667.

Alzaatreh, A., Lee, C. and Famoye, F. (2013), A new method for generating families of continuous distributions. Metron, 71(1), 63-79. 
Anderson, T. W. (2011), Anderson-darling tests of goodness-of-fit. International Encyclopedia of Statistical Science (pp. 52-54), Springer Berlin Heidelberg.

Arnold, B. C., Balakrishnan, N. and Nagaraja, H. N. (2008), A first course in order statistics. Society for Industrial and Applied Mathematics.

Aryal, G. R. and Tsokos, C. P. (2011), Transmuted Weibull distribution: A generalization of the Weibull probability distribution. European Journal of Pure and Applied Mathematics, 4(2), 89-102.

Bourguignon, M., Silva, R. B. and Cordeiro, G. M. (2014), The Weibull-G family of probability distributions. Journal of Data Science, 12(1), 53-68.

Cordeiro, G. M., Alizadeh, M., Ozel, G., Hosseini, B., Ortega, E. M. M. and Altun, E. (2017), The generalized odd log-logistic family of distributions: properties, regression models and applications. Journal of Statistical Computation and Simulation, 87(5), 908932.

Eugene, N., Lee, C. and Famoye, F. (2002), The beta-normal distribution and its applications. Communications in Statistics - Theory and Methods, 31(4), 497-512.

Korkmaz, M. Ç. and Gen, A. İ. (2017), A new generalized two-sided class of distributions with an emphasis on two-sided generalized normal distribution. Communications in Statistics - Simulation and Computation, 46(2), 1441-1460.

Lee E. T. and Wang J. W. (2003), Statistical methods for survival data analysis, 3rd edition, Wiley, NewYork.

Marshall, A. W. and Olkin, I. (1997), A new method for adding a parameter to a family of distributions with application to the exponential and Weibull families. Biometrika. 84, 641-652.

Mirhossaini, S. M. and Dolati, A. (2008), On a new generalization of the exponential distribution. Journal of Mathematical Extension, Journal of Mathematical Extension, 3(1), 27-42.

Nadarajah, S., Cordeiro, G. M. and Ortega, E. M. (2013), The exponentiated Weibull distribution: a survey. Statistical Papers, 54(3), 839-877.

Nofal, Z. M., Afify, A. Z., Yousof, H. M. and Cordeiro, G. M. (2017), The generalized transmuted-G family of distributions. Communications in Statistics - Theory and Methods, 46(8), 4119-4136. 
Pogány, T. K., Saboor, A. and Provost, S. (2015), The Marshall-Olkin exponential Weibull distribution. Hacettepe Journal of Mathematics and Statistics, 44(6),1579.

Rousu, D. N. (1973). Weibull skewness and kurtosis as a function of the shape parameter. Technometrics, 927-930.

Shaw, W. T. and Buckley, I. R. C. (2007), The alchemy of probability distributions: Beyond Gram-Charlier \& Cornish-Fisher expansions, and skew-normal or kurtoticnormal distributions. Research report.

Singh, S. K., Singh, U. and Sharma, V. K. (2013), Bayesian analysis for Type- II hybrid censored sample from inverse Weibull distribution. International Journal of System Assurance Engineering and Management, 4(3), 241-248.

Stephens, M. A. (1979), The Anderson-Darling statistic (No. TR-39). Stanford University, Department of Statistics.

Yousof, H. M., Afify, A. Z., Alizadeh, M., Butt, N. S., Hamedani, G. G. and Ali, M. M. (2015), The transmuted exponentiated generalized-G family of distributions. Pakistan Journal of Statistics and Operation Research, 11(4), 441-464. 


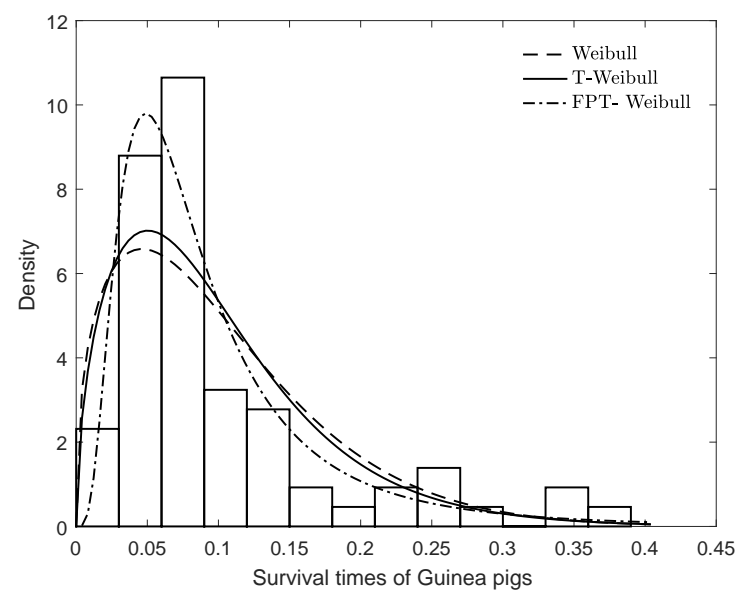

Figure 7: The histogram and the pdfs' of the fitted models for Guinea pigs data.
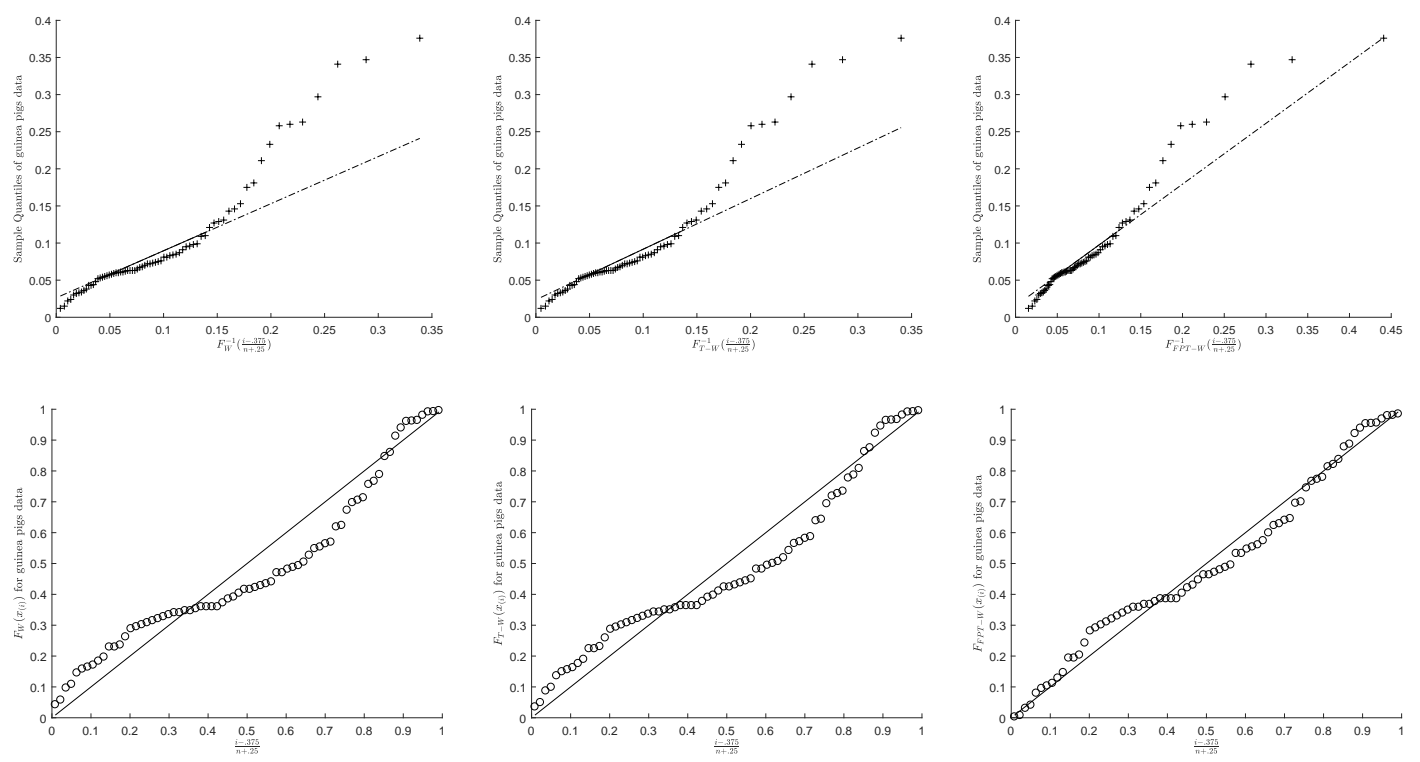

Figure 8: Q-Q and P-P plots of Weibull, Transmuted-Weibull and FPT-Weibull for Guinea pigs data. 
Table 6: The remission times (in months) of bladder cancer patients.

\begin{tabular}{llllcccllc}
\hline 0.08 & 2.09 & 3.48 & 4.87 & 6.94 & 8.66 & 13.11 & 23.63 & 0.20 & 2.23 \\
0.52 & 4.98 & 6.97 & 9.02 & 13.29 & 0.40 & 2.26 & 3.57 & 5.06 & 7.09 \\
0.22 & 13.80 & 25.74 & 0.50 & 2.46 & 3.64 & 5.09 & 7.26 & 9.47 & 14.24 \\
0.82 & 0.51 & 2.54 & 3.70 & 5.17 & 7.28 & 9.74 & 14.76 & 26.31 & 0.81 \\
0.62 & 3.82 & 5.32 & 7.32 & 10.06 & 14.77 & 32.15 & 2.64 & 3.88 & 5.32 \\
0.39 & 10.34 & 14.83 & 34.26 & 0.90 & 2.69 & 4.18 & 5.34 & 7.59 & 10.66 \\
0.96 & 36.66 & 1.05 & 2.69 & 4.23 & 5.41 & 7.62 & 10.75 & 16.62 & 43.01 \\
0.19 & 2.75 & 4.26 & 5.41 & 7.63 & 17.12 & 46.12 & 1.26 & 2.83 & 4.33 \\
0.66 & 11.25 & 17.14 & 79.05 & 1.35 & 2.87 & 5.62 & 7.87 & 11.64 & 17.36 \\
0.40 & 3.02 & 4.34 & 5.71 & 7.93 & 11.79 & 18.10 & 1.46 & 4.40 & 5.85 \\
0.26 & 11.98 & 19.13 & 1.76 & 3.25 & 4.50 & 6.25 & 8.37 & 12.02 & 2.02 \\
0.31 & 4.51 & 6.54 & 8.53 & 12.03 & 20.28 & 2.02 & 3.36 & 6.76 & 12.07 \\
0.73 & 2.07 & 3.36 & 6.93 & 8.65 & 12.63 & 22.69 & 5.49 & & \\
\hline
\end{tabular}

Table 7: MLEs of the model parameters and values of goodness of fit statistics (bladder cancer patients' data).

\begin{tabular}{|c|c|c|c|c|c|}
\hline Model & Parameter Estimates & K-S & AIC & $\mathcal{A}^{2}$ & $W^{2}$ \\
\hline Weibull & $\hat{\sigma}=9.5607 \quad \hat{\eta}=1.0478$ & 0.0700 & 832.17 & 0.9577 & 0.1537 \\
\hline T- Weibull & $\begin{array}{c}\hat{\sigma}=14.6198 \quad \hat{\eta}=1.1333 \\
\hat{\lambda}=0.7449\end{array}$ & 0.0588 & 829.92 & 0.5600 & 0.0879 \\
\hline $\begin{array}{c}\text { FPT- } \\
\text { Weibull }\end{array}$ & $\begin{array}{ll}\hat{\sigma}=8.1605 & \hat{\eta}=0.7770 \\
\hat{\lambda}=1.6423 & \hat{k}=2.1319\end{array}$ & 0.0421 & 828.40 & 0.2033 & 0.0299 \\
\hline
\end{tabular}




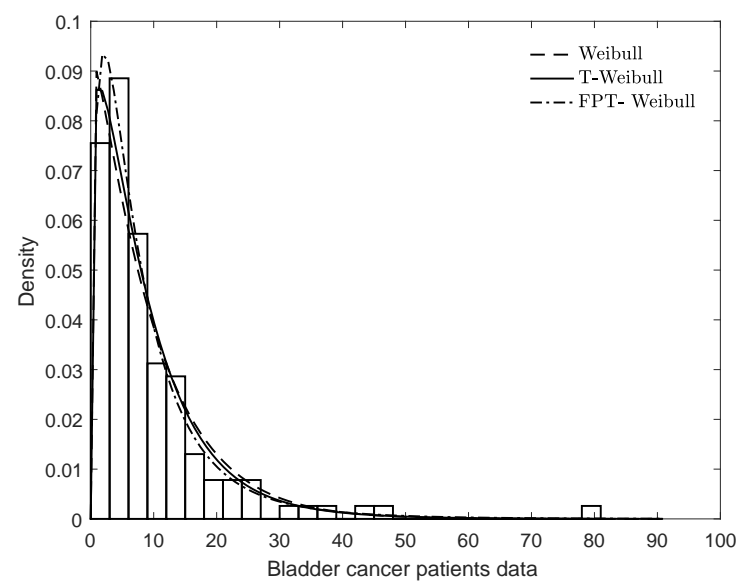

Figure 9: The histogram and the pdfs' of the fitted models for bladder cancer patients' data.
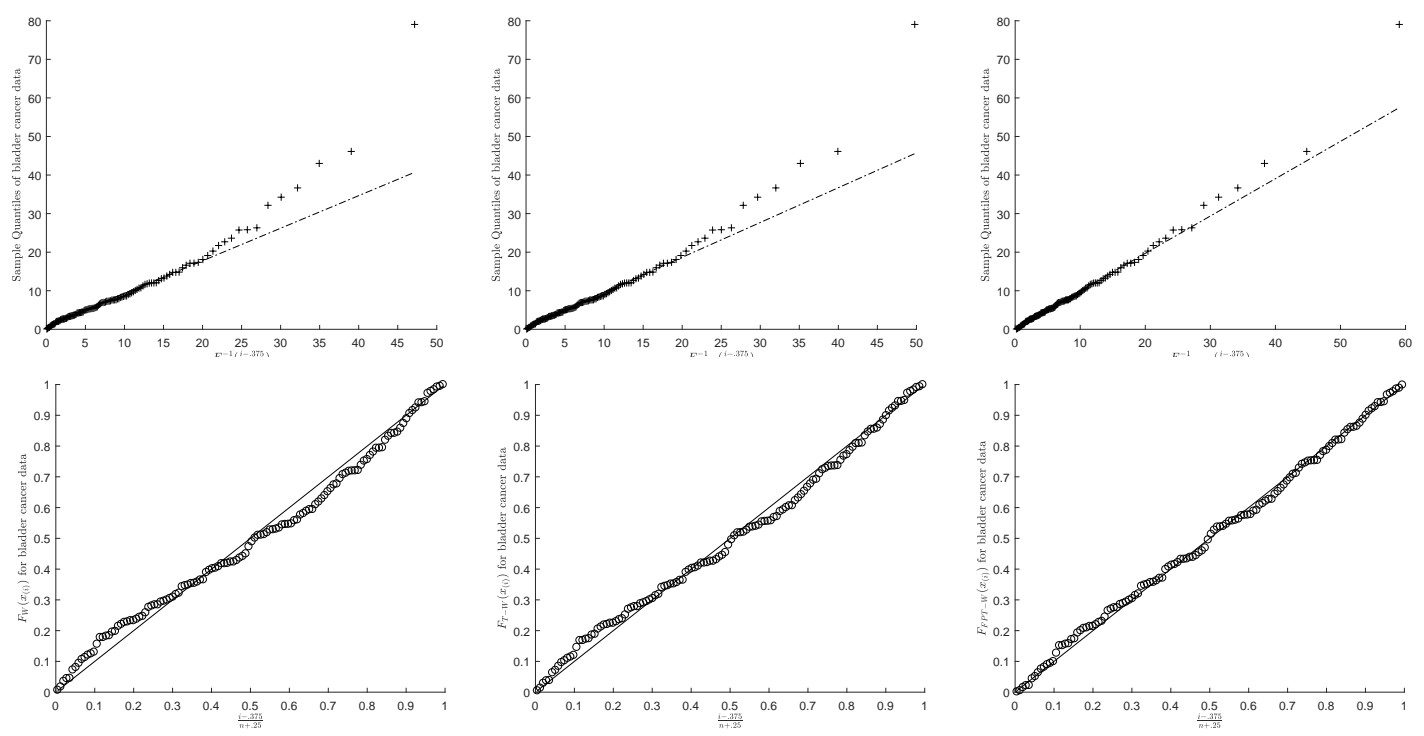

Figure 10: Q-Q and P-P plots of Weibull, Transmuted-Weibull and FPT-Weibull for bladder cancer patients' data 